Research Article

\title{
INVESTIGATION OF SHADE TREE SPECIES USED IN TEA GARDEN IN BANGLADESH
}

\author{
M.A. Rahman ${ }^{1 *}$, Z.R. Moni' ${ }^{2}$ M.A. Rahman ${ }^{3}$ and S. Nasreen ${ }^{1}$ \\ ${ }^{1}$ Forest Protection Division, Bangladesh Forest Research Institute \\ Chattogram-4000, Bangladesh \\ ${ }^{2}$ Senior Scientific Officer, Bangladesh Agricultural Research Council (BARC) \\ Farmgate, Dhaka 1215, Bangladesh \\ ${ }^{3}$ Seed Orchard Division, Bangladesh Forest Research Institute, Chattogram-4000, Bangladesh
}

\begin{abstract}
The shade trees are an integral component of tea cultivation in Bangladesh. The shade trees are essential for modulating the environment of the tea ecosystem, enriching the soil fertility, reducing temperature and the evaporative capacity, conserve soil moisture and helps in the control of certain pests and diseases which are positively thermotropic in nature. The shade trees provide partial shade to the tea plants, which is important for improving the quality of the tea leaf. The right type of shade trees and their proper management is a prerequisite for successful tea crop growing. For this persists, a floristic exploration of shade trees was carried out at fifteen tea gardens in Chattogram and Moulvibazar District of Bangladesh from November 2017 to December 2018. During the investigation, a total of 44 species of Angiosperm representing 31 genera of 9 families was enlisted. For every species, scientific name, vernacular name, status, and necessary photographs are mentioned. In the assessment, the Fabaceae family shows the highest number of shade trees comprising 19 genera and 31 species. The most common permanent shade tree species among the tea gardens are Albizia odoratissima, A. chinensis, A. lebbeck, A. lucidior, A. procera, and Derris robusta. Indigofera teysmannii is frequently using as a temporary shade species in all investigated tea gardens. Cajanus cajan, Tephrosia candida, Tephrosia candida, Gliricidia sepium, Erythrina lithosperma and Desmodium gyroides species are also used as temporary shade trees in many tea gardens. Further investigations, however,are required to find out the right type of shade tree species on the growth and yield of tea plants in tea cultivation areas of Bangladesh.
\end{abstract}

Keywords: Tea, Tea garden, Shade tree species, Fabaceae

\footnotetext{
**Corresponding author: bappy43@yahoo.com
} 


\section{INTRODUCTION}

Tea (Camellia sinensis L.) is one of the most popular and favored beverages (nonalcoholic) in the world. It plays an important role in Bangladesh economy. There are 162 tea estates and 746 small cultivators having about 59,018 hectares of tea plantation generating about 85.05 million $\mathrm{kg}$ of over tea per annum with an average yield of about 1,587 kg per hectare in Bangladesh (BTB, 2017). It is now ranked 8th, $10^{\text {th }}$, and $12^{\text {th }}$ position in the world in respect of the area, production, and export respectively (ITC, 2015). The tea industry of Bangladesh is frolicking a major role in fulfilling the domestic consumption as well as an important source of export earnings and contributing about 0.8 percent of the total GDP of the country (Rahman, 2016). Tea gardens and smallholding tea cultivators in Bangladesh have created employment for about 1,33,000 people every year (Rahman, 2016).

The tea plant is traditionally grown under the shade condition as they are shadeloving plants. Planting of shade trees has become an integral component of tree plantation and management in India, Sri Lanka, Indonesia, Bangladesh and some parts of Africa. The shade trees provide $50 \%$ to $70 \%$ of diffused solar insolation to the tea cultivation area (Sana, 1989). It results in improves the quality of the tea leaves due to an increase in the concentration of amino acids with lowers the content of catechin in the plant and it also inhibits the concentration of flavonoid (Ku et al., 2010; Wang et al., 2012). Barua (1979) reported that tree shade can increase photosynthetic rate and yield by 10 to 30 percent, especially during hot summer in north-eastern India. Shade trees not only used as shade provider, but it also produces fuel wood timber, conserve soil from erosion, the impact of rainfall drop, enrich soil fertility, support diverse flora and fauna, producing foods (leaves, pods or flowers) for people, creation of tannins, gums, medicines and services like living fences, ornamentals and environmental protection (FAO, 1987).

Tea cultivation is one of the important Agroforestry systems well known in Bangladesh, which was started in the $19^{\text {th }}$ century.The shade trees play an important role in increasing the productivity of the tea under the environment of Bangladesh. Without shade trees, the yield of tea is limited. Thus, to increase tea yields, a large number of shade tree species are planted in various tea gardens of Bangladesh (Kalita et al., 2014). The right type of shade trees and their proper management is possibly

one of the most well-investigated research problems in all tea cultivation areas of Bangladesh. Therefore, the present study was undertaken to investigate the shade tree species in the tea gardens of Bangladesh.

\section{MATERIALS AND METHODS}

\section{Study area}

The present study was conducted in fifteen tea gardens of Chattogram and Moulvibazar District of Bangladesh (Table 1). This study was conducted in several tea estates from September 2017 to December 2018. 
Table 1. Investigated tea estates in Bangladesh

\begin{tabular}{|c|c|c|c|}
\hline SL no. & Name of tea garden & Location & District \\
\hline 1. & Ramgarh tea estate & Fatikchari & Chattogram \\
\hline 2. & Dantmara tea estate & Bhojpur, Fatikchari & Chattogram \\
\hline 3. & BTRI substation & Ooadalia, Fatikchari & Chattogram \\
\hline 4. & Oodalia tea estate & Katirhat, Fatikchari & Chattogram \\
\hline 5. & Bhojpur tea estate & Bhojpur, Fatikchari & Chattogram \\
\hline 6. & Naseha tea estate & Dantmara, Fatikchari & Chattogram \\
\hline 7. & Haldavalley tea estate & Najirhat, Fatikchari & Chattogram \\
\hline 8. & Neapchun tea estate & Narayanhat, Fatikchari & Chattogram \\
\hline 9. & Panchabati tea estate & Bhojpur, Fatikchari & Chattogram \\
\hline 10. & Aasia tea estate & Bhojpur, Fatikchari & Chattogram \\
\hline 11. & Majan tea estate & Datmara, Fatikchari & Chattogram \\
\hline 12. & Andhar manik tea estate & Chikancherra, Fatikchari & Chattogram \\
\hline 13. & BTRI main campus & Srimangal & Maulvibazar \\
\hline 14. & $\begin{array}{l}\text { BTRI Bilashchara } \\
\text { Experimental Farm }\end{array}$ & Srimangal & Maulvibazar \\
\hline 15. & Finlay tea estate & Srimangal & Maulvibazar \\
\hline
\end{tabular}

\section{Specimen collection and plant identification}

During the study, a large number of fertile specimens of different shade trees have been collected for taxonomic identification. The collected specimens were identified consulting with the experts of (Bangladesh Forest Research Institute) BFRI Herbarium (BFRIH) and pertinent literature (Ahmed et al., 2009; Pasha and Uddin, 2013; Hooker, 1872-1897; Prain, 1903; Brandis 1906; Kanjilal et al.,1939, 1940; Heining, 1925). Also, we compared identified samples with specimens preserved at Bangladesh Forest Research Institute Herbarium (BFRIH) Chattogram, Bangladesh.

\section{RESULTS AND DISCUSSION}

During the present investigation, mainly two types of shade trees viz., permanent shade trees, and temporary shade trees were found in all investigated tea gardens of Bangladesh. Permanent shade trees are planted for a longer period (about 40 years). It takes a long period to be established with their full canopy. That's why at the initial stage of plantation temporary shade trees along with the permanent shade trees are planted to protect the tea plants from direct sunlight. When the permanent shade trees become established after 5/6 years, the temporary shade trees are removed. In the present assessment, a total of 44 species representing 31 genera of 9 families of angiosperms were enlisted (Table 2). The Morphological characteristics of shade tree species at investigated tea estates are presented at Table 3. 
Table 2. Types and abundance of shade trees at investigated tea estates

\begin{tabular}{|c|c|c|c|c|c|}
\hline $\begin{array}{l}\text { Scientific } \\
\text { Name }\end{array}$ & Tea garden name & Habits & $\begin{array}{l}\text { Vernacular } \\
\text { Name }\end{array}$ & Family & Status \\
\hline \multicolumn{6}{|c|}{ Permanent shade trees } \\
\hline $\begin{array}{l}\text { Acacia } \\
\text { auriculiformis }\end{array}$ & RMG, DM, BP, HV, NPC,AS, ADM & Tree & Akashmoni & Fabaceae & $\begin{array}{l}\text { Less } \\
\text { common }\end{array}$ \\
\hline A. hybrid & $\mathrm{BP}, \mathrm{MJ}, \mathrm{DM}$ & Tree & $\begin{array}{l}\text { Acacia } \\
\text { hybrid }\end{array}$ & Fabaceae & Rare \\
\hline $\begin{array}{l}\text { Albizia } \\
\text { chinensis }\end{array}$ & $\begin{array}{l}\text { RMG, DM, BTRISS, ODL, BP, } \\
\text { NDFC, HV, NPC, PACB, AS, MJ, } \\
\text { ADM, BTRIMCS, BEFBTRIS, FLA }\end{array}$ & Tree & Chakua koroi & Fabaceae & $\begin{array}{l}\text { Most } \\
\text { common }\end{array}$ \\
\hline A. lebbeck & $\begin{array}{l}\text { RMG, DM, BTRISS, ODL, BP, } \\
\text { NDFC, HV, NPC, PACB, AS, MJ, } \\
\text { ADM, BTRIMCS, BEFBTRIS, FLA }\end{array}$ & Tree & Sirish & Fabaceae & $\begin{array}{l}\text { Most } \\
\text { common }\end{array}$ \\
\hline A. lucidor & $\begin{array}{l}\text { RMG, DM, BTRISS, ODL, BP, } \\
\text { NDFC, HV, NPC, PACB, AS, MJ, } \\
\text { ADM, BTRIMCS, BEFBTRIS, FLA }\end{array}$ & Tree & Potka Siris & Fabaceae & $\begin{array}{l}\text { Most } \\
\text { common }\end{array}$ \\
\hline $\begin{array}{l}\text { Acacia } \\
\text { mangium }\end{array}$ & BP, HV, NPC, PACB & Tree & Mangium & Fabaceae & Rare \\
\hline $\begin{array}{l}\text { Albizia } \\
\text { moluccana }\end{array}$ & $\begin{array}{l}\text { RMG, BP, NDFC, HV, NPC, } \\
\text { PACB, AS, MJ, ADM }\end{array}$ & Tree & Moluccan sau & Fabaceae & $\begin{array}{l}\text { Less } \\
\text { Common }\end{array}$ \\
\hline A. procera & $\begin{array}{l}\text { RMG, DM, BTRISS, ODL, BP, } \\
\text { NDFC, HV, NPC, PACB, AS, MJ, } \\
\text { ADM, BTRIMCS, BEFBTRIS, FLA }\end{array}$ & Tree & Silkoroi & Fabaceae & $\begin{array}{l}\text { Most } \\
\text { common }\end{array}$ \\
\hline A. richardiana & ODL,NPC, AS, MJ & Tree & Gagan shiris & Fabaceae & Rare \\
\hline A. falcataria & $\begin{array}{l}\text { RMG, DM, ODL, BP, NDFC, HV, } \\
\text { NPC, AS, ADM, BEFBTRIS, FLA }\end{array}$ & Tree & $\begin{array}{l}\text { Malakana } \\
\text { Koroi }\end{array}$ & Fabaceae & $\begin{array}{l}\text { Less } \\
\text { Common }\end{array}$ \\
\hline $\begin{array}{l}\text { Adenanthera } \\
\text { pavonina }\end{array}$ & $\begin{array}{l}\text { RMG, ODL, BP, HV, NPC, AS, MJ, } \\
\text { ADM }\end{array}$ & Tree & $\begin{array}{l}\text { Rakta } \\
\text { Kambal }\end{array}$ & Fabaceae & $\begin{array}{l}\text { Less } \\
\text { common }\end{array}$ \\
\hline $\begin{array}{l}\text { Albizia } \\
\text { odoratissima }\end{array}$ & $\begin{array}{l}\text { RMG, DM, BTRISS, ODL, BP, } \\
\text { NDFC, HV, NPC, PACB, AS, MJ, } \\
\text { ADM, BTRIMCS, BEFBTRIS, FLA }\end{array}$ & Tree & Kali Sirish & Fabaceae & $\begin{array}{l}\text { Most } \\
\text { common }\end{array}$ \\
\hline $\begin{array}{l}\text { Alstonia } \\
\text { scholaris }\end{array}$ & $\begin{array}{l}\text { RMG, DM, ODL, BP, HV, PACB, } \\
\text { AS, MJ, ADM }\end{array}$ & Tree & Chatim & $\begin{array}{l}\text { Apocynace } \\
\text { ae }\end{array}$ & $\begin{array}{l}\text { Less } \\
\text { common }\end{array}$ \\
\hline $\begin{array}{l}\text { Artocarpus } \\
\text { chaplasha }\end{array}$ & DM, ODL & Tree & Chapalish & Moraceae & Rare \\
\hline $\begin{array}{l}\text { Azadirachta } \\
\text { indica }\end{array}$ & $\begin{array}{l}\text { RMG, DM, ODL, BP, NDFC, HV, } \\
\text { NPC, PACB, AS, MJ, ADM }\end{array}$ & Tree & Neem & Meliaceae & $\begin{array}{l}\text { Less } \\
\text { common }\end{array}$ \\
\hline $\begin{array}{l}\text { Bauhinia } \\
\text { purpurea }\end{array}$ & DM, ODL, BP, AS, MJ & Tree & $\begin{array}{l}\text { Rakta } \\
\text { kanchan }\end{array}$ & Fabaceae & Rare \\
\hline $\begin{array}{l}\text { Butea } \\
\text { monosperma }\end{array}$ & DM, ODL, AS & Tree & Palash & Fabaceae & Rare \\
\hline Cassia fistula & RMG, BP, NDFC, HV, NPC, AS, & Tree & Badar lathi & Fabaceae & Less \\
\hline
\end{tabular}




\begin{tabular}{|c|c|c|c|c|c|}
\hline $\begin{array}{l}\text { Scientific } \\
\text { Name }\end{array}$ & Tea garden name & Habits & $\begin{array}{l}\text { Vernacular } \\
\text { Name }\end{array}$ & Family & Status \\
\hline & MJ, ADM & & & & common \\
\hline Cassia siamea & $\mathrm{BP}, \mathrm{NDFC}, \mathrm{HV}, \mathrm{AS}$ & Tree & Minjiri & Fabaceae & Rare \\
\hline $\begin{array}{l}\text { Chukrasia } \\
\text { tabularis }\end{array}$ & $\mathrm{DM}, \mathrm{BP}, \mathrm{AS}$ & Tree & Chikrassi & Meliaceae & Rare \\
\hline $\begin{array}{l}\text { Dalbergia } \\
\text { assamica }\end{array}$ & RMG, DM, NPC & Tree & Mouhita & Fabaceae & Rare \\
\hline $\begin{array}{l}\text { Dalbergia } \\
\text { sericea }\end{array}$ & $\begin{array}{l}\text { RMG, DM, ODL, BP, HV, NPC, } \\
\text { PACB, AS, MJ, ADM }\end{array}$ & Tree & $\begin{array}{l}\text { Silky } \\
\text { Dalbergia }\end{array}$ & Fabaceae & $\begin{array}{l}\text { Less } \\
\text { common }\end{array}$ \\
\hline $\begin{array}{l}\text { Dalbergia } \\
\text { sissoo }\end{array}$ & $\begin{array}{l}\text { RMG, ODL, BP, , NPC, PACB, AS, } \\
\text { MJ, ADM }\end{array}$ & Tree & Shisu & Fabaceae & $\begin{array}{l}\text { Less } \\
\text { common }\end{array}$ \\
\hline Delonix regia & $\mathrm{DM}, \mathrm{BP}, \mathrm{AS}, \mathrm{MJ}$ & Tree & Gulmohar & Fabaceae & Rare \\
\hline Derris robusta & $\begin{array}{l}\text { RMG, DM, BTRISS, ODL, BP, } \\
\text { NDFC, HV, NPC, PACB, AS, MJ, } \\
\text { ADM, BTRIMCS, BEFBTRIS, FLA }\end{array}$ & Tree & Miringa & Fabaceae & $\begin{array}{l}\text { Most } \\
\text { common }\end{array}$ \\
\hline $\begin{array}{l}\text { Erythrina } \\
\text { variegata }\end{array}$ & RMG, ODL, HV & Tree & Madar & Fabaceae & Rare \\
\hline $\begin{array}{l}\text { Eucalyptus } \\
\text { robusta }\end{array}$ & DM, ODL, AS & Tree & Eucalyptus & Myrtaceae & Rare \\
\hline $\begin{array}{l}\text { Eucalyptus } \\
\text { camaldulensis }\end{array}$ & $\mathrm{DM}, \mathrm{ODL}$ & Tree & Red gum & Myrtaceae & Rare \\
\hline $\begin{array}{l}\text { Gravillea } \\
\text { robusta }\end{array}$ & RMG, DM, ODL, BP & Tree & Silky oak & Proteaceae & Rare \\
\hline $\begin{array}{l}\text { Lagerstroemia } \\
\text { speciosa }\end{array}$ & $\mathrm{RMG}, \mathrm{BP}, \mathrm{AS}, \mathrm{MJ}$ & Tree & Jarul & Lythraceae & Rare \\
\hline $\begin{array}{l}\text { Leucaena } \\
\text { leucocephalla }\end{array}$ & $\begin{array}{l}\text { DM, ODL, NDFC, HV, NPC, AS, } \\
\text { MJ, ADM }\end{array}$ & Tree & Ipilipil & Fabaceae & $\begin{array}{l}\text { Less } \\
\text { common }\end{array}$ \\
\hline $\begin{array}{l}\text { Mangifera } \\
\text { indica L. }\end{array}$ & RMG, BP, AS & Tree & Aam & $\begin{array}{l}\text { Anacardiac } \\
\text { eae }\end{array}$ & Rare \\
\hline $\begin{array}{l}\text { Melia } \\
\text { azedarachta }\end{array}$ & $\mathrm{DM}, \mathrm{ODL}, \mathrm{BP}, \mathrm{AS}$ & Tree & Ghora neem & Meliaceae & Rare \\
\hline $\begin{array}{l}\text { Phyllanthus } \\
\text { emblica }\end{array}$ & DM, ODL & Tree & Amlaki & $\begin{array}{l}\text { Phyllanthac } \\
\text { eae }\end{array}$ & Rare \\
\hline $\begin{array}{l}\text { Samanea } \\
\text { saman }\end{array}$ & DM, ODL & Tree & $\begin{array}{l}\text { Shirish/Rain } \\
\text { tree }\end{array}$ & Fabaceae & Rare \\
\hline $\begin{array}{l}\text { Swietenia } \\
\text { macrophylla }\end{array}$ & RMG, BTRISS, ODL, BP, MJ, ADM & Tree & Mahagoni & Meliaceae & Rare \\
\hline Toona ciliata & AS, MJ & Tree & Tun & Meliaceae & Rare \\
\hline \multicolumn{6}{|c|}{ Temporary shade trees } \\
\hline $\begin{array}{l}\text { Indigofera } \\
\text { teysmannii }\end{array}$ & $\begin{array}{l}\text { RMG, DM, BTRISS, ODL, BP, } \\
\text { NDFC, HV, NPC, PACB, AS, MJ, } \\
\text { ADM, BTRIMCS, BEFBTRIS, FLA }\end{array}$ & Shrubs & Indigofera & Fabaceae & $\begin{array}{l}\text { Most } \\
\text { common }\end{array}$ \\
\hline
\end{tabular}




\begin{tabular}{|c|c|c|c|c|c|}
\hline $\begin{array}{l}\text { Scientific } \\
\text { Name }\end{array}$ & Tea garden name & Habits & $\begin{array}{l}\text { Vernacular } \\
\text { Name }\end{array}$ & Family & Status \\
\hline Cajanus cajan & $\begin{array}{l}\text { RMG, DM, ODL, BP, NDFC, HV, } \\
\text { NPC, PACB, AS, MJ, ADM, }\end{array}$ & Shrubs & Arhor & Fabaceae & Common \\
\hline $\begin{array}{l}\text { Tephrosia } \\
\text { candida }\end{array}$ & $\mathrm{AS}, \mathrm{MJ}$ & Shrubs & $\begin{array}{l}\text { White } \\
\text { tephrosia }\end{array}$ & Fabaceae & Rare \\
\hline $\begin{array}{l}\text { Gliricidia } \\
\text { sepium }\end{array}$ & $\begin{array}{l}\text { RMG, DM, ODL, BP, NDFC, HV, } \\
\text { NPC, AS, MJ, ADM }\end{array}$ & Shrubs & Gliricidia & Fabaceae & $\begin{array}{l}\text { Less } \\
\text { common }\end{array}$ \\
\hline $\begin{array}{l}\text { Erythrina } \\
\text { lithosperma }\end{array}$ & RMG, ODL & $\begin{array}{l}\text { Small } \\
\text { tree }\end{array}$ & Dadap & Fabaceae & Rare \\
\hline $\begin{array}{l}\text { Desmodium } \\
\text { gyroides }\end{array}$ & RMG, BP, NPC & $\begin{array}{l}\text { Mediu } \\
\text { m size } \\
\text { tree }\end{array}$ & Tick clover & Fabaceae & Rare \\
\hline
\end{tabular}

RMG- Ramgarh; DM-Dantmara; BTRISS- BTRI substation, Fatikchari; ODL- Oodalia; BP- Bhojpur; NDFC- Naseha; HV- Haldavalley; NPC- Neapchun;PACB- Panchabati; AS- Aasia; MJ- Majan; ADMAndhar manik;BTRIMCS- BTRI Main campus, Srimangal; BEFBTRIS-Bilashchara Experimental Farm, BTRI, Srimangal; FLA- Finlay tea estate, Srimangal.

Table 3. Morphological characteristics of shade tree species at investigated tea estates

Shade trees species

Akashmoni (Acacia auriculiformis)
A medium-sized, heavily branched, evergreen tree that grows between to $15-30 \mathrm{~m}$ tall. Bark greyish brown, more or less smooth in young trees. Adults phyllodes alternate, straight or falcate, acute or subacute, $10-15 \mathrm{~cm}$ long and $1.2-2.5 \mathrm{~cm}$ wide, glabrous. Flowers in a spike up to $7.5 \mathrm{~cm}$ long, many in pairs in the upper leaf-axils, golden colored. Fruit a pod, initially straight, but maturity become very much irregularly twisted and coiled. Seed elliptical, 3-5 x 2-3 mm, almost encircled by a long orange funicle.

Acacia hybrid (A. The tree is a medium-sized. The tree is capable of reaching a height of hybrid)

Chakua koroi (Albizia chinensis) $8-10 \mathrm{~m}$ and a diameter at breast height of 7.5-9.0 $\mathrm{cm}$. Phyllode is about 4-6 cm wide and 15-20 cm long, with four veins. The flowers are creamy to whitish and arranged in a straight, or slightly bent, 8-10 $\mathrm{cm}$ spike. The pod (fruit) is usually very curly and twists. A pod holds 5-9 seeds (Fig. 4d).

The tree is a deciduous or evergreen. Bark dark brown or greenishgrey with many vertical and horizontal fissures. Leaves bipinnate, rachis $7-30 \mathrm{~cm}$ long with a large gland near the base and sometimes one or more between the pinnae. Its flowers are stalked heads that aggregate into a yellow panicle. Flowers are sessile, yellowish white in pedunculated, terminal or axillary heads. The fruits are indehiscent pods (Fig. 3 e). 


\begin{tabular}{|c|c|}
\hline $\begin{array}{l}\text { Shade trees } \\
\text { species }\end{array}$ & Morphological characteristics \\
\hline $\begin{array}{l}\text { Sirish }(A . \\
\text { lebbeck) }\end{array}$ & $\begin{array}{l}\text { The tree can attain a height of } 30 \mathrm{~m} \text { and a diameter of } 1 \mathrm{~m} \text {. Bark } \\
\text { yellowish grey to dark brown, almost blackish. Leaves bipinnate } \\
\text { rachis } 7-15 \mathrm{~cm} \text { long, usually with a large gland near the base or } \\
\text { between the upper pinnae. Flowers greenish white, very fragrant in } \\
\text { peduncled heads, solitary or in fascicles of } 2-4 \text { from the upper leaf } \\
\text { axils. Fruit a pod, } 20-30 \text { x } 2.5-5 \mathrm{~cm} \text {, very compressed and flat. Seeds } \\
4-12 \text { (Fig. } 3 \text { a). }\end{array}$ \\
\hline $\begin{array}{l}\text { Potka Siris (A. } \\
\text { lucidor) }\end{array}$ & $\begin{array}{l}\text { A medium sized to large unarmed, glabrous evergreen or semi- } \\
\text { deciduous tree with spreading branches. This tree species grows up to } \\
40 \text { m. Leaves dark green, bi-pinnae. The leaves are (single) pinnate, in } \\
\text { 1-3 pairs. The seed pods are yellow and glabrous. Seeds } 6-8 \text {, round } \\
\text { (Fig. } 3 \mathrm{f} \text { ). }\end{array}$ \\
\hline $\begin{array}{l}\text { Mangium } \\
\text { (Acacia } \\
\text { mangium) }\end{array}$ & $\begin{array}{l}\text { It is a fast-growing evergreen tree with a dense, spreading crown. The } \\
\text { bole is usually straight, often fluted near the base, free of branches. } \\
\text { phyllode large, thicky coriaceous, up to } 25 \mathrm{~cm} \text { long and } 5-10 \mathrm{~cm} \\
\text { broad, whitish or yellowshish green, glabrous. Flowers creamy white } \\
\text { in a loose spike up to } 10 \mathrm{~cm} \text { long. Fruits is a pod, blackish brown and } \\
\text { woody. }\end{array}$ \\
\hline $\begin{array}{l}\text { Moluccan sau } \\
\text { (Albizia } \\
\text { moluccana) }\end{array}$ & $\begin{array}{l}\text { It is about } 30 \mathrm{~m} \text { tall tree in nature with a massive trunk and an open } \\
\text { crown. Leaves are twice pinnately compound with small leaflets. } \\
\text { Flowers are creamy white small flowers are faintly fragrant. Fruits are } \\
\text { pods that fall from the trees when mature (Fig. } 4 \text { a). }\end{array}$ \\
\hline $\begin{array}{l}\text { Silkoroi }(A . \\
\text { procera })\end{array}$ & $\begin{array}{l}\text { A large deciduous tree with tall cylindrical boles and rather small } \\
\text { crown. Bark yellow shish brown to greenish white. Leaves bipinnate, } \\
\text { pinnae 3-5 pairs, } 12-25 \mathrm{~cm} \text { long. Flowers sessile, yellowish white, in } \\
\text { fascicled heads of } 2-5 \text {, sometimes solitary, arrange in terminal and } \\
\text { axillary pinnacles. Fruits a pod, flat, flexible, } 7-12 \times 1.2-2.5 \mathrm{~cm} \text {. Seeds } \\
6-12 \text { (Fig. 3c). }\end{array}$ \\
\hline $\begin{array}{l}\text { Gagan shiris }(A . \\
\text { richardiana) }\end{array}$ & $\begin{array}{l}\text { A lofty hand some evergreen tree with horizontal dichotomous } \\
\text { branching which from a beautiful crown. Bark yellowish gray. Leaves } \\
\text { bipinnate, 6-9 cm long, pinnae } 8-14 \text {, stipules small. Flowers small, } \\
\text { greenish white, sessile in axillary corymbose, much shorter than } \\
\text { leaves. Fruits a pod, dull brownish grey, firm, flat about } 10 \times 2 \mathrm{~cm} \text {, } \\
\text { shortly beaked (Fig. 4b). }\end{array}$ \\
\hline $\begin{array}{l}\text { Malakana } \\
\text { Koroi }(A . \\
\text { falcataria })\end{array}$ & $\begin{array}{l}\text { Tall deciduous tree to } 30 \mathrm{~m} \text { tall, } 1 \mathrm{~m} \text { in diameter. Leaves alternate, } \\
\text { bipinnate, } 23-30 \mathrm{~cm} \text { long, rufose pubescent, the pinnae } 20-24,5-10 \\
\mathrm{~cm} \text { long, each with } 30-40 \text { paired leaflets, sessile, obliquely oblong, } 6- \\
12 \mathrm{~mm} \text { long, } 3-5 \mathrm{~mm} \text { broad, shortly acute. Flowers sessile, white, ca } \\
10-12 \mathrm{~mm} \text { long. Pods } 10-13 \mathrm{~cm} \text { long, } 2 \mathrm{~cm} \text { wide, flat, acute, green, } \\
\text { turning brown, papyraceous, dehiscent. Seeds } 15-20 \text { per pod. }\end{array}$ \\
\hline
\end{tabular}




\begin{tabular}{|c|c|}
\hline $\begin{array}{l}\text { Shade trees } \\
\text { species }\end{array}$ & Morphological characteristics \\
\hline $\begin{array}{l}\text { Rakta Kambal } \\
\text { (Adenanthera } \\
\text { pavonina) }\end{array}$ & $\begin{array}{l}\text { A medium sized, unarmed, deciduous tree. Bark brown or greyish } \\
\text { brown, corky. Leaves bipinnate, rachis } 20-25 \mathrm{~cm} \text { long, red; pinnae 4-6 } \\
\text { pairs, } 7-8 \mathrm{~cm} \text { long, opposite; leaflets alternate, 12-20, elliptic, obtuse, } \\
\text { base unequal, dark green and glabrous above. Flowers in long panicled } \\
\text { racemes, greenish yellow, fragrant, minute. Fruit a pod, 15-24 x } 1.5 \\
\mathrm{~cm} \text {, flat, linear, curved and much twisted when opening. Seeds 8-15. }\end{array}$ \\
\hline $\begin{array}{l}\text { Kali Sirish } \\
\text { (Albizia } \\
\text { odoratissima) }\end{array}$ & $\begin{array}{l}\text { A large semi deciduous or semi evergreen tree with spreading crown. Bark } \\
\text { thick, rough yellowish brown. Leaves bi-pinnate, rachis up to } 30 \mathrm{~cm} \text { long } \\
\text { with 3-8 pairs of pinnae and has a large gland at the base and } 1-2 \text { between } \\
\text { the upper pinnae. Flowers small, sessile, yellowish in heads of compact } \\
\text { corymbs, arranged in large terminal panicles. Fruit a pod, } 15-20 \times 2.5 \mathrm{~cm} \text {, } \\
\text { dehiscent, thin, flexible, tomentose. Seed } 8-12 \text { (Fig. } 3 \mathrm{~d} \text { ). }\end{array}$ \\
\hline $\begin{array}{l}\text { Chatim } \\
\text { scholari }\end{array}$ & $\begin{array}{l}\text { A medium sized evergreen trees with copious white latex, trunk often } \\
\text { flutted; branches whorled and twigs with white streaks of lenticles } \\
\text { which are containing. Bark grey and smooth. Leaves in whorls of } 4-8 \text {, } \\
\text { oblancelate or elliptic-oblong. Flowers greenish white in compact } \\
\text { umbellately branched pubescent panicled cymes. Fruits of follicles, 30- } \\
60 \mathrm{~cm} \text { long, pendulous, in clusters, terete. }\end{array}$ \\
\hline $\begin{array}{l}\text { Chapalish } \\
\text { (Artocarpus } \\
\text { chaplasha) }\end{array}$ & $\begin{array}{l}\text { A large deciduous tree with tall cylindrical bole and milky latex. Bark } \\
\text { thick, greyish brown or ashy-gray with large white patches deep } \\
\text { verticle furrows peeling off in rounded flakes. Leaves are juvenile, } \\
\text { elliptic ovate } 15-20 \mathrm{~cm} \text { long, hispid, base-subcordate or rounded. } \\
\text { Flowers monoecious, densely crowded on globose receptacles. Fruits a } \\
\text { globes, tuberculate, syncarp, pubescent (Fig. } 4 \mathrm{c} \text { ). }\end{array}$ \\
\hline $\begin{array}{l}\text { Neem } \\
\text { (Azadirachta } \\
\text { indica) }\end{array}$ & $\begin{array}{l}\text { A medium sized to large glabrous evergreen to semi deciduous tree with } \\
\text { large spreading crown. Bark greyish to blackish color. Leaves } \\
\text { imparipinnate, } 18-45 \mathrm{~cm} \text { long, crowded towards the end of branchlets. } \\
\text { Flowers short, white, honey-scented, pentamerous, in axillary panicles } \\
\text { shorten than leaves. Fruit a drupe, } 1-15 \mathrm{~cm} \text { long, ovoid, pericarp smooth. }\end{array}$ \\
\hline $\begin{array}{l}\text { Rakta Kanchan } \\
\text { (Bauhinia } \\
\text { purpurea) }\end{array}$ & $\begin{array}{l}\text { A small to medium sized, evergreen to semi evergreen tree. Leaves } \\
\text { deeply cleft, leaf cleft halfway or more down. Flowers very fragrant, } \\
\text { deep rose or lilac. Fruits a pod, } 15-30 \mathrm{~cm} \text { long, flat. Seeds 12-15, } \\
\text { flattened, roundish, dark brown, smooth. }\end{array}$ \\
\hline $\begin{array}{l}\text { Palash (Butea } \\
\text { monosperma) }\end{array}$ & $\begin{array}{l}\text { A small to medium size tree. Bark rough, bluish-grey, exfoliating in } \\
\text { irregular pieces; inner bark fibirous, pinkish, exuding reddish juice } \\
\text { when injured. Leaves pinnately } 3 \text {-foliate, common petiole } 12-25 \mathrm{~cm} \\
\text { long, geniculate, pubescent, stipules short, tomentose. Flowers large, } \\
\text { reddish-orange, in rigid axillary racemes, } 10-15 \mathrm{~cm} \text { long and crowded } \\
\text { towards the ends of leafless branchlets. Fruits a pod, } 10-15 \times 3-4 \mathrm{~cm} \text {, } \\
\text { rigid, pendulous and borne in large clusters in the leafless branches. } \\
\text { Seed oval, compressed, dark brown. }\end{array}$ \\
\hline
\end{tabular}




\begin{tabular}{|c|c|}
\hline $\begin{array}{l}\text { Shade trees } \\
\text { species }\end{array}$ & Morphological characteristics \\
\hline $\begin{array}{l}\text { Badar lathi } \\
\text { (Cassia fistula) }\end{array}$ & $\begin{array}{l}\text { A small to medium sized deciduous tree, often with crooked bole. } \\
\text { Young shoots and leaves silky. Bark greenish to ash grey. Leaves } \\
\text { pinnate, } 24-40 \mathrm{~cm} \text { long; rachis slightly hairy; stipule minute, hairy, } \\
\text { early falling; Flowers yellow, in pendulous, lax, axillary, } 20-60 \mathrm{~cm} \\
\text { long racemes. Fruit a pod, } 30-60 \mathrm{~cm} \text { long and about } 25 \mathrm{~cm} \text { across, } \\
\text { calendric, indehiscent, smooth, dark brown to black when ripe. Seeds } \\
40-100 \text {, flattened, ovoid, about } 1 \mathrm{~cm} \text { across, embedded in a dark brown } \\
\text { or black sweetish pulp. }\end{array}$ \\
\hline $\begin{array}{l}\text { Minjiri (Cassia } \\
\text { siamea) }\end{array}$ & $\begin{array}{l}\text { A medium size to large, fast growing, evergreen or semi deciduous } \\
\text { tree. Bark ash grey or blackish brown. Leaves pari-pinnate, } 15-30 \mathrm{~cm} \\
\text { long. Flowers Sulphur colored, in stout corymbose racemes arranged } \\
\text { in a large pyramidal terminal panicles, often } 60 \mathrm{~cm} \text { long. Fruit a pod, } \\
\text { flat, ribbon like, thickened at sutures, } 10-25 \mathrm{~cm} \text { long; greenish brown } \\
\text { velvety when mature. Seeds many, pentagonally elliptical, flat, } \\
\text { blackish brown, glossy. }\end{array}$ \\
\hline $\begin{array}{l}\text { Chikrassi } \\
\text { (Chukrasia } \\
\text { tabularis) }\end{array}$ & $\begin{array}{l}\text { A tall, handsome (due to beautiful foliage), deciduous tree with tall } \\
\text { cylindrical bole and spreading crown; branchlets lenticellate. Bark } \\
\text { thick, rusty brown. Leaves pinnately compound, } 30-50 \mathrm{~cm} \text { long; softly } \\
\text { pubescent when young, rachis terete. Flowers white in terminal, } \\
\text { panicles, shorten than leaves, slightly hairy. Fruit a capsule, ellipsoidal, } \\
\text { woody, brown or blackish outside, speckled with white lenticels. Seeds } \\
\text { numerous, transversely and closely packed, elliptic, flat with dark } \\
\text { brown wings. }\end{array}$ \\
\hline $\begin{array}{l}\text { Mouhita } \\
\text { (Dalbergia } \\
\text { assamica) }\end{array}$ & $\begin{array}{l}\text { D. assamica is a tree with a spreading crown; it can grow } 7-10 \text { metres } \\
\text { tall. Branches horizontally spreading. Leaves } 25-30 \mathrm{~cm} \text {; stipules } \\
\text { caducous, large, leaflike, ovate to ovate-lanceolate; leaflets } 13- \\
21 \text {; Flowers } 6-8 \mathrm{~mm} \text {. Legume broadly ligulate or oblong to strap- } \\
\text { shaped, } 5-9 \times 1.2-1.8(-2.5) \mathrm{cm} \text {, leathery, base attenuate, cuneate, apex } \\
\text { acute, inconspicuously reticulate opposite } 1 \text { or } 2(-4) \text { seeds. Seeds } \\
\text { reniform, compressed, ca. } 6 \times 2.5 \mathrm{~mm} \text {. }\end{array}$ \\
\hline $\begin{array}{l}\text { Silky Dalbergia } \\
\text { (Dalbergia } \\
\text { sericea) }\end{array}$ & $\begin{array}{l}\text { Dalbergia is a medium to large-sized trees, } 40-60 \mathrm{~m} \text { tall; trunk up to } 2 \\
\mathrm{~m} \text {. Leaves are imparipinnate, alternate, } 14-22 \mathrm{~cm} \text { long. Flowers are } \\
\text { borne in silky-hairy, dense-flowered panicles, } 3-4 \mathrm{~cm} \text { long, in leaf } \\
\text { axils. Pods are elliptic-oblong or oblong, } 2.2-6 \times 0.5-0.7 \mathrm{~cm} \text {, acute- } \\
\text { obtuse at tip, tapering towards base into a } 3-4 \mathrm{~mm} \text { long slender stalk, } \\
\text { flat, glabrous, reticulately nerved especially on seed portion. }\end{array}$ \\
\hline $\begin{array}{l}\text { Shisu (Dalbergia } \\
\text { sissoo) }\end{array}$ & $\begin{array}{l}\text { A medium sized to very large deciduous tree often with curved bole or } \\
\text { crooked bole. Bark thick, grey or light brown, recticulately and } \\
\text { longitudinally furrowed. Leaves imparipinnate, } 10-20 \mathrm{~cm} \text { long, rachis } \\
\text { zigzag. Flowers yellowish white, in } 2.5-4.0 \mathrm{~cm} \text { long cymose racemes. } \\
\text { Fruit a pod, strap-shaped, pale brown, } 5.8 \times 0.7-1.2 \mathrm{~cm} \text {. Seeds flat, } \\
\text { kidney shaped. }\end{array}$ \\
\hline
\end{tabular}




\begin{tabular}{|c|c|}
\hline $\begin{array}{l}\text { Shade trees } \\
\text { species }\end{array}$ & Morphological characteristics \\
\hline $\begin{array}{l}\text { Gulmohar } \\
\text { (Delonix regia) }\end{array}$ & $\begin{array}{l}\text { A medium sized handsome, deciduous or evergreen tree with } \\
\text { spreading, dome shaped or flat-top crown. Leaves bi-pinnate, 15-40 } \\
\text { cm long, pinnae } 11-18 \text { pairs. Flowers large, } 7-10 \mathrm{~cm} \text { across, scarlet. } \\
\text { Fruit a sessile pod, } 30-60 \mathrm{~cm} \text { long, flat, linear, obliquely acuminate, } \\
\text { woody when dry but dehiscent. Seeds many, oblong, slightly } \\
\text { compressed, about } 1.5 \mathrm{~cm} \text { long; brown and white variegated, bony } \\
\text { when dry. }\end{array}$ \\
\hline $\begin{array}{l}\text { Miringa (Derris } \\
\text { robusta) }\end{array}$ & $\begin{array}{l}\text { A medium sized deciduous tree with tall cylindrical bole and } \\
\text { dichotomous branching at the top. Bark greyish white. Leaves } \\
\text { pinnately compound, } 7-20 \mathrm{~cm} \text { long. Flowers white in selender axillary } \\
\text { pubescent racemes, } 12-25 \mathrm{~cm} \text { long; pedicles filiform, grey downy. } \\
\text { Fruits a pod, } 2.5-7 \times 3 \mathrm{~cm} \text {, linear, narrowed at both end. Seeds brown, } \\
\text { orbicular, compressed (Fig. } 3 \mathrm{~b} \text { ). }\end{array}$ \\
\hline $\begin{array}{l}\text { Madar } \\
\text { variega }\end{array}$ & $\begin{array}{l}\text { A small to medium sized armed, deciduous tree. Bark smooth, } \\
\text { yellowish or greenish grey, peeling off in thin. Leaves tri foliate, } \\
\text { common petiole } 15-20 \mathrm{~cm} \text { long, unarmed, terete. Flowers showy, } \\
\text { scarlet red, in } 15-25 \mathrm{~cm} \text { long racemes. Fruit a pod, } 10-12 \mathrm{~cm} \text { long, } \\
\text { cylindrical with constrictions. Seeds kidney shaped, large, deep red. }\end{array}$ \\
\hline $\begin{array}{l}\text { Eucalyptus } \\
\text { (Eucalyptus } \\
\text { robusta) }\end{array}$ & $\begin{array}{l}\text { It is a medium to large tree with a dense crown and long, spreading } \\
\text { branches when grown in open ground. The bark is rough and persistent } \\
\text { to the small branches, thick, held in coarse, soft, spongy, elongated } \\
\text { slabs with deep longitudinal furrows, grey or reddish grey-brown. The } \\
\text { juvenile leaves are petiolate, ovate, up to } 19 \times 8 \mathrm{~cm} \text {, strongly } \\
\text { discolorous, green, opposite for several pairs, then alternate. The } \\
\text { inflorescence is axillary, 9-15 flowered, the peduncles are strongly } \\
\text { flattened, up to } 3 \mathrm{~cm} \text { in length. The fruit is a woody capsule, with } \\
\text { prominent stalk, cylindrical, } 1.8 \times 1.1 \mathrm{~cm}\end{array}$ \\
\hline $\begin{array}{l}\text { Red gum } \\
\text { (Eucalyptus } \\
\text { camaldulensis) }\end{array}$ & $\begin{array}{l}\text { A large boled, medium sized to tall evergreen tree. Bark smooth white, } \\
\text { grey, buff, reddish patches. Leaves bluish green; juvenile alternate, } \\
\text { petiolate, broad lanceolate. Flowers in axillary, } 5-10 \text { flowered umbels } \\
\text { on a slender, terate, } 0.5-2.5 \mathrm{~cm} \text { long peduncles. Fruits a capsule, } \\
\text { pedicillate, ovoid or tuncate globular, } 0.3-0.6 \times 0.4-1 \mathrm{~cm} \text { disc broad. } \\
\text { Seeds smooth yellow. }\end{array}$ \\
\hline $\begin{array}{l}\text { Silky oak } \\
\text { (Gravillea } \\
\text { robusta) }\end{array}$ & $\begin{array}{l}\text { A medium sized to tall, semi deciduous tree with pyramidal or conical } \\
\text { crown. Bark dark grey to blackish, furrowed, lenticellete. Leaves } \\
\text { alternate, pinnately compound, petiolate, graceful and fin like, } \\
\text { pinnatifid, divided with } 11-23 \text { primary segments. Flowers bright } \\
\text { orange or orange yellow glabrous, in one sided } 7-10 \mathrm{~cm} \text { long racemes } \\
\text { and borne in dense cluster on short leafless branches. Fruit boat } \\
\text { shadeped } 2 \text { seeded follicles, compressed and oblique and tipped with a } \\
\text { selender persistent style. Seed brown, flat with a shiny centre } \\
\text { surrounded by a light brown papery wing. }\end{array}$ \\
\hline
\end{tabular}




\begin{tabular}{|c|c|}
\hline $\begin{array}{l}\text { Shade trees } \\
\text { species }\end{array}$ & Morphological characteristics \\
\hline $\begin{array}{l}\text { Jarul } \\
\text { (Lagerstroemia } \\
\text { speciose) }\end{array}$ & $\begin{array}{l}\text { A medium sized to large much branched deciduous tree. Bark greyish } \\
\text { to brown, smooth, peeling off in thin irregular flakes, blaze whitish. } \\
\text { Leaves opposite, elliptic or oblong-lanceolate, } 10-20 \mathrm{~cm} \text { long, } \\
\text { acuminate, glabrous on both surface. Flowers large, showy, mauve } \\
\text { purple, } 5-7 \mathrm{~cm} \text { across, in ample terminal panicles, ultimate branches } \\
\text { mostly 1-3 flowered. Fruit a capsule, sub globes, smooth, seated on } \\
\text { accrescent woody. Seeds pale brown, } 3 \text { angular, laterally expanded } \\
\text { into an oblong wing. }\end{array}$ \\
\hline $\begin{array}{l}\text { Ipilipil } \\
\text { (Leucaena } \\
\text { leucocephalla) }\end{array}$ & $\begin{array}{l}\text { A medium sized to tall, large, unarmed, deciduous tree. Bark light } \\
\text { brownish grey, smooth. Leaves bipinnate, } 7-18 \mathrm{~cm} \text { long; pinnae } 8-16 \\
\mathrm{~cm} \text { long; leaflets } 20-30 \text {, about } 12 \mathrm{~cm} \text { long, glaucous, linear, acute, } \\
\text { finely hairy. Flowers dirty white or yellowish in auxillary or sub } \\
\text { terminal, dense globose heads. Fruit a pod, produced very copiously, } \\
\text { straight, } 7-15 \times 1.0-1.4 \mathrm{~cm} \text { flat, glabrous, shiny brown when mature. } \\
\text { Seeds } 15-20 \text {, lenticular, shiny. }\end{array}$ \\
\hline $\begin{array}{l}\text { Aam (Mangifera } \\
\text { indica L.) }\end{array}$ & $\begin{array}{l}\text { A medium-sized to large evergreen tree with a large dense crown and } \\
\text { rather short buttressed trunk. Bark brown or ashy-grey, vertically } \\
\text { cracked, exfoliating in scales. Leaves crowded at the end of branchlets, } \\
\text { oblong-lanceolate, } 15-20 \mathrm{~cm} \text { long, blunty acuminate, coriaceous, } \\
\text { glossy, green above, paler beneath, entire with a undulated margin. } \\
\text { Flowers in erect pubescent panicle. Fruit a drupe, ovoid or globose, } \\
\text { laterally compressed. }\end{array}$ \\
\hline $\begin{array}{l}\text { Ghora neem } \\
\text { (Melia } \\
\text { azedarachta) }\end{array}$ & $\begin{array}{l}\text { A medium-sized to large glabrous evergreen to semi-deciduous trees } \\
\text { with large spreading crown. Bark greyish brown to blackish with } \\
\text { longitudinal and oblique furrows and many scattered tubercles, blaze } \\
\text { greenish pink with characteristic smell. Leaves imparipinnate, } 18-45 \\
\mathrm{~cm} \text { long, crowded towards the end of branchlets. Flowers short, white, } \\
\text { honey-scented, pentamerous, in auxillary panicles shorter than leaves. } \\
\text { Fruit a drupe, } 1-1.5 \mathrm{~cm} \text { long, ovoid, pericarp smooth, greenish yellow } \\
\text { when ripe enclosing white visid pulp and a smooth whitish stony } \\
\text { endocarp almost } 1 \text {-seeded. }\end{array}$ \\
\hline $\begin{array}{l}\text { Amlaki } \\
\text { (Phyllanthus } \\
\text { emblica) }\end{array}$ & $\begin{array}{l}\text { A small to medium sized deciduous tree. Bark of young tree grey- } \\
\text { smooth, in older trees exfoliating in irregular rounded pieces and in } \\
\text { long strips. Leaves not compound, although individual small leaves } \\
\text { look very much like the leaflets of pinnately compound leaf, light } \\
\text { green, bluntish, } 0.6-1.3 \mathrm{~cm} \text { long, glabrous, paler beneath with apprised } \\
\text { pubescence, closely arranged in two rows on opposite side of twigs. } \\
\text { Flowers small, greenish yellow, monoecious, in axillary clusters, } \\
\text { usually crowded towards the lower naked portion of branchlets, male } \\
\text { flowers on short, slender pedicles; female flowers sub-sessile. Fruit a } \\
\text { drupe, globose, about } 2.5 \mathrm{~cm} \text { across, obscurely 6-lobed, } 3 \text { celled. Seeds } \\
\text { dark brown, trigonous. }\end{array}$ \\
\hline
\end{tabular}




\begin{tabular}{|c|c|}
\hline $\begin{array}{l}\text { Shade trees } \\
\text { species }\end{array}$ & Morphological characteristics \\
\hline $\begin{array}{l}\text { Shirish/Rain tree } \\
\text { (Samanea } \\
\text { saman) }\end{array}$ & $\begin{array}{l}\text { A very large, deciduous to semi-deciduous tree with spreading crown } \\
\text { and large bole. Bark rough, blackish-grey, exfoliated in old trees. } \\
\text { Leaves bipinnate, pinnae } 3-7 \text { pairs, largest uppermost, shorter } \\
\text { downwards with a gland between each pair of pinnae. Flowers in dense } \\
\text { rose colored heads on pubescent peduncles, } 1-3 \text { together from the } \\
\text { upper axils, and } 5-8 \mathrm{~cm} \text { long. Fruit a pod, } 12-20 \times 1.5 \mathrm{~cm} \text {. indehiscent, } \\
\text { suture thick, ripe pod blackish brown, smooth with septa between } \\
\text { seeds and containing sweet sticky pulp. }\end{array}$ \\
\hline $\begin{array}{l}\text { Mahagoni } \\
\text { (Swietenia } \\
\text { macrophylla) }\end{array}$ & $\begin{array}{l}\text { A tall evergreen to semi deciduous tree. Bark greyish brown, rough } \\
\text { and flakes off in small patches. Leaves paripinnate, up to } 60 \mathrm{~cm} \text { long; } \\
\text { leaflets } 6-16 \text {, ovate-lanceolate with oblique base, up to } 20 \mathrm{~cm} \text { long } \\
\text { with a very short panicles. Fruit a woody capsule, erect, brown. Seeds } \\
\text { deep brown, winged at one end only. }\end{array}$ \\
\hline $\begin{array}{l}\text { Tun (Toona } \\
\text { ciliata) }\end{array}$ & $\begin{array}{l}\text { A medium to large deciduous tree. Bark greyish brown, exfoliating in } \\
\text { irregular flakes when old. Leaves paripinnate, } 30-60 \mathrm{~cm} \text { long, } \\
\text { somewhat crowded at the ends of branchlets; common petiole, } \\
\text { glabrous, terete. Flowers white, sweet scented, in terminal pyramidal } \\
\text { cymose panicle. Fruit a capsule, dark brown, smooth outside with } \\
\text { white lenticles. Seed brown with lighter brownish wing at the ends. }\end{array}$ \\
\hline $\begin{array}{l}\text { Indigofera } \\
\text { (Indigofera } \\
\text { teysmannii) }\end{array}$ & $\begin{array}{l}\text { Erect shrub or small tree, up to } 12 \mathrm{~m} \text { tall. Branches subsericeous with } \\
\text { minute brown or white, biramous, appressed hairs. Leaves } \\
\text { imparipinnate; stipules linear, up to } 8 \mathrm{~mm} \text { long; leaflets } 11-23 \text {, } \\
\text { elliptical to ovate, } 2-8 \mathrm{~cm} \times 1-3 \mathrm{~cm} \text {. Inflorescence an axillary, many- } \\
\text { flowered raceme, } 8-10 \mathrm{~cm} \text { long; flowers about } 0.5 \mathrm{~cm} \text { long; calyx } \\
\text { brown-sericeous, } 2 \mathrm{~mm} \text { long; corolla whitish, pink or dark purple; } \\
\text { standard ovate, up to } 5 \mathrm{~mm} \times 4 \mathrm{~mm} \text {, dorsally sericeous. Pod } \\
\text { subcylindrical, } 2.5-4.5 \mathrm{~cm} \times 0.5 \mathrm{~cm} \text {, glabrous, beaked, about 16- } \\
\text { seeded. I. zollingeriana occurs mainly on coral strands and sandy } \\
\text { beaches, up to } 850 \mathrm{~m} \text { altitude (Fig. } 4 \mathrm{e} \text { ). }\end{array}$ \\
\hline $\begin{array}{l}\text { Arhor(Cajanus } \\
\text { cajan) }\end{array}$ & $\begin{array}{l}\text { The plant is an erect, short-lived perennial leguminous shrub that } \\
\text { usually grows to a height of about } 1-2 \mathrm{~m} \text {, but can reach up to } 2-5 \mathrm{~m} \\
\text { high. It quickly develops a deep }(2 \mathrm{~m} \text { depth) poisonous taproot. The } \\
\text { stems are woody at the base, angular and branching. The leaves are } \\
\text { alternate and trifoliate. The leaflets are oblong and lanceolate, } 5-10 \mathrm{~cm} \\
\text { long } \times 2-4 \mathrm{~cm} \text { wide. Leaves and stems are pubescent. The flowers }(5 \text { to } \\
\text { 10) are grouped in racemes at the apices or axils of the branches. The } \\
\text { flowers are papilionaceous and generally yellow in colour. They can } \\
\text { also be striated with purple streaks. The corolla is about } 2-2.5 \mathrm{~cm} \text {. The } \\
\text { fruit is a flat, straight and pubescent pod, } 5-9 \mathrm{~cm} \text { long } \times 12-13 \mathrm{~mm} \\
\text { wide. It contains } 2-9 \text { seeds that are brown, red or black in colour, small } \\
\text { and sometimes hard-coated. }\end{array}$ \\
\hline
\end{tabular}




\section{Shade trees} species

White
tephrosia(Tephro
sia candida)

Gliricidia

(Gliricidia sepium)

Dadap (Erythrina lithosperma)

Tick clover (Desmodium gyroides)
Morphological characteristics

Tephrosia candida is a deciduous Shrub growing to $3 \mathrm{~m}(9 \mathrm{ft})$ by $3 \mathrm{~m}$ (9ft) at a medium rate. Leaves alternate, 17-25-foliolate; rachis 15-25 $\mathrm{cm}$, including petiole; leaflets oblong, 3-6 × 0.6-1.4 cm, abaxially densely sericeous, adaxially glabrous, secondary veins $30-50$ on each side of midvein. Flowers ca. $2 \mathrm{~cm}$. Calyx ca. $5 \times 5 \mathrm{~mm}$; teeth equal, ca. $1 \mathrm{~mm}$, apex rounded. Petals white, rarely yellow or pale pink; standard densely sericeous. Ovary tomentose, with numerous ovules. Fruit linear, $8-10 \mathrm{~cm} \times 7.5-8.5 \mathrm{~mm}$, straight, brown tomentose with a mixture of long and short trichomes, apex truncate and with a straight ca. $1 \mathrm{~cm}$ beak; seeds $10-15$ per legume, olive-green with dark patches, ellipsoid, ca. $5 \times 3.5 \mathrm{~mm}$, smooth.

A small to medium-sized, deciduous tree with a short trunk and long slender branches at first tend to rise almost vertically from the base. Bark soft, grey or ashy with longitudinal cracks. Bark of branches brownish-grey speckled with white spots like lenticels. Leaves imparipinnate, to $30 \mathrm{~cm}$ long. Flowers mauve pinkish; produced in great clustered racemes on leafless branches. Fruit a pod, to $20 \times 2 \mathrm{~cm}$, flat without wing and dehiscent seeds up to 10 or more (Fig. $4 \mathrm{f}$ ).

The plant is deciduous growing to $20 \mathrm{~m}(65 \mathrm{ft})$ by $20 \mathrm{~m}(65 \mathrm{ft})$ at a fast rate. The branches and the branchlets stout and armed with short, few to many sharp prickles. Leaflets are broadly ovate and 8 to 18 centimeters long, with pointed tip and broad base. Racemes are terminal, hairy, dense, and up to 2.5 centimeters long. Flowers are papillonaceous, large and numerous. Calyx is about 4 centimeters long and minutely 5-toothed at the tip, the mouth being very oblique. Petals are bright red and shorter than the calyx, the standard being 7 to 9 centimeters long and the wings and keels subequal. Stamens are 10, upper filaments free nearly to the base or more or less connate with others. Ovary many-ovuled, style incurved. Racemes terminal, hairy, dense and up to $2.5 \mathrm{~cm}$ long. Fruits are pods, 10 to 25 centimeters long, 1.5 to 2 centimeters in diameter, and distinctly constricted between the seeds.

Leaves are compound. Leaflets are hairy, more densely hairy along major veins on the underside, to 3 inches long and 1 inch wide with a rounded or slightly tapering base and blunt point at the tip. Flowers are pea-shaped, about $1 / 2$ inch long, pink to purple with 2 yellow spots near the base of the broad upper lobe. The stamens and pistil form a curving tube that protrudes from the center, between the 2 lateral petals. The calyx behind the flower and the short flower stalk are reddish green and hairy. Fruit is a flat pod 1 to $2 \frac{1}{2}$ inches long with 3 to 5 sections, the sections convex on the upper edge and well rounded on the lower, and each containing a single seed. Seeds are kidney-shaped, about 4 $\mathrm{mm}$ long, and mature to brown. The pod is densely covered in tiny hooked hairs that latch onto anything that passes by. 
Among the collected species, Fabaceae shows the highest percentage with 31 species comprising 19 genera (61.29\%) followed by 5 species of Meliaceae comprising 5 genera (16.12\%) and 2 species of Myrtaceae comprising 1 genus (3.22\%) (Fig. 1 and 2). Apocynaceae, Moraceae, Protaceae, Lythraceae, Anacardiaceae, and Phyllanthaceae each family show the one species of one genera (Fig. 1 and 2).

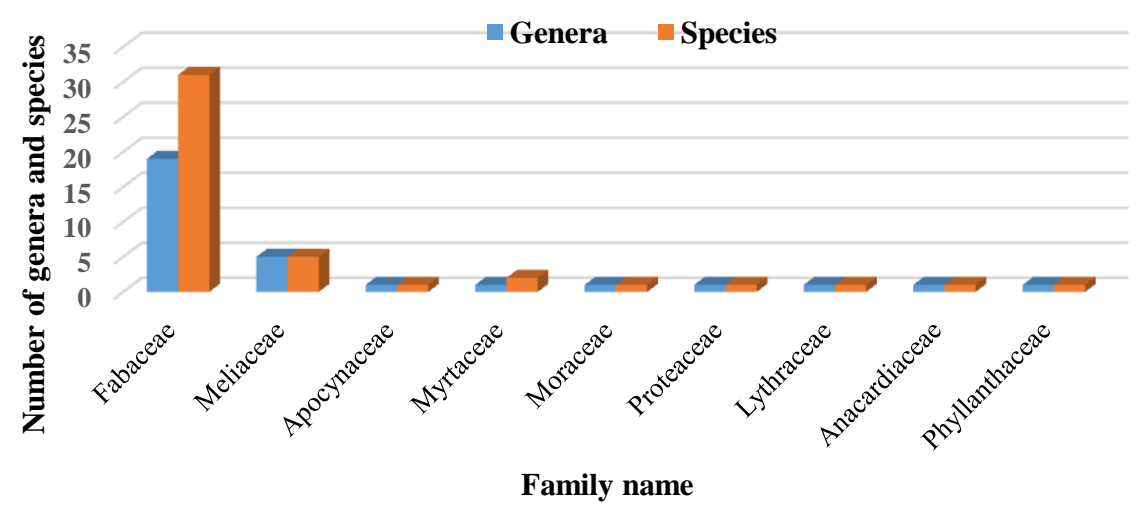

Figure 1. Family wise showing the number of genera and speciesof shade trees at the surveyed tea garden.

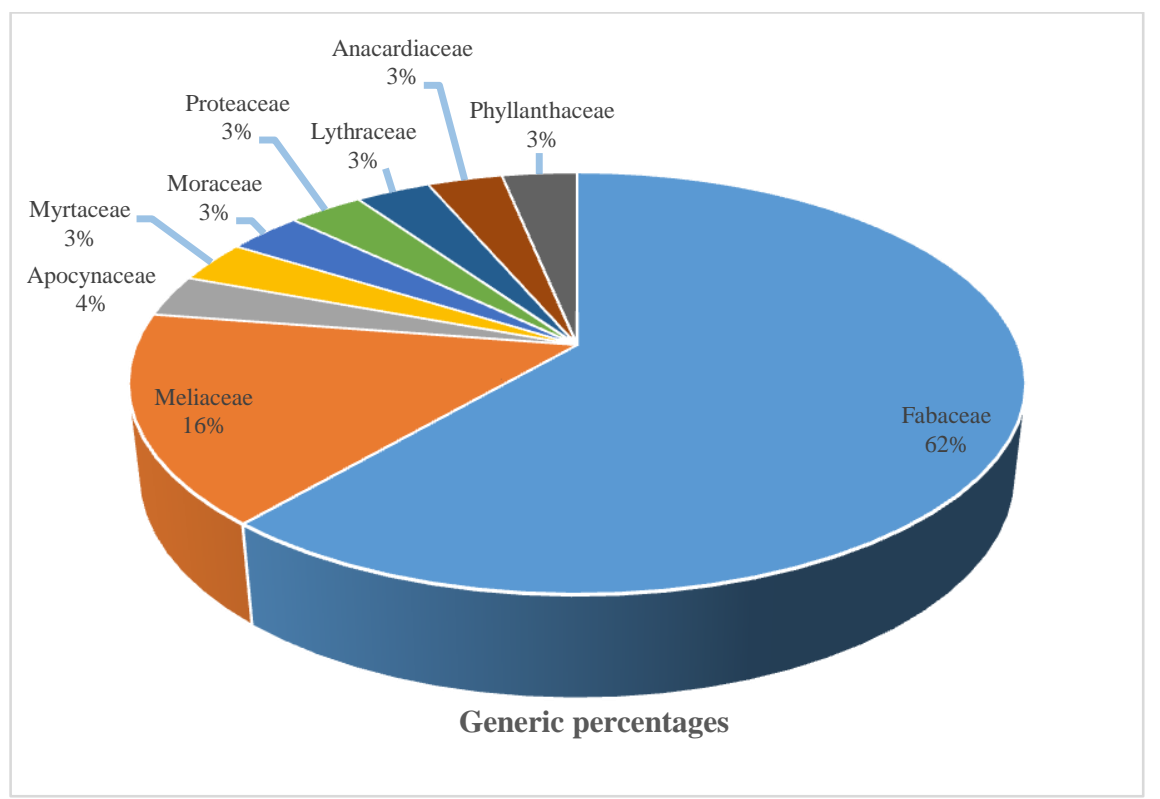

Figure 2. Family wise generic percentage of available shade trees of the study areas. 

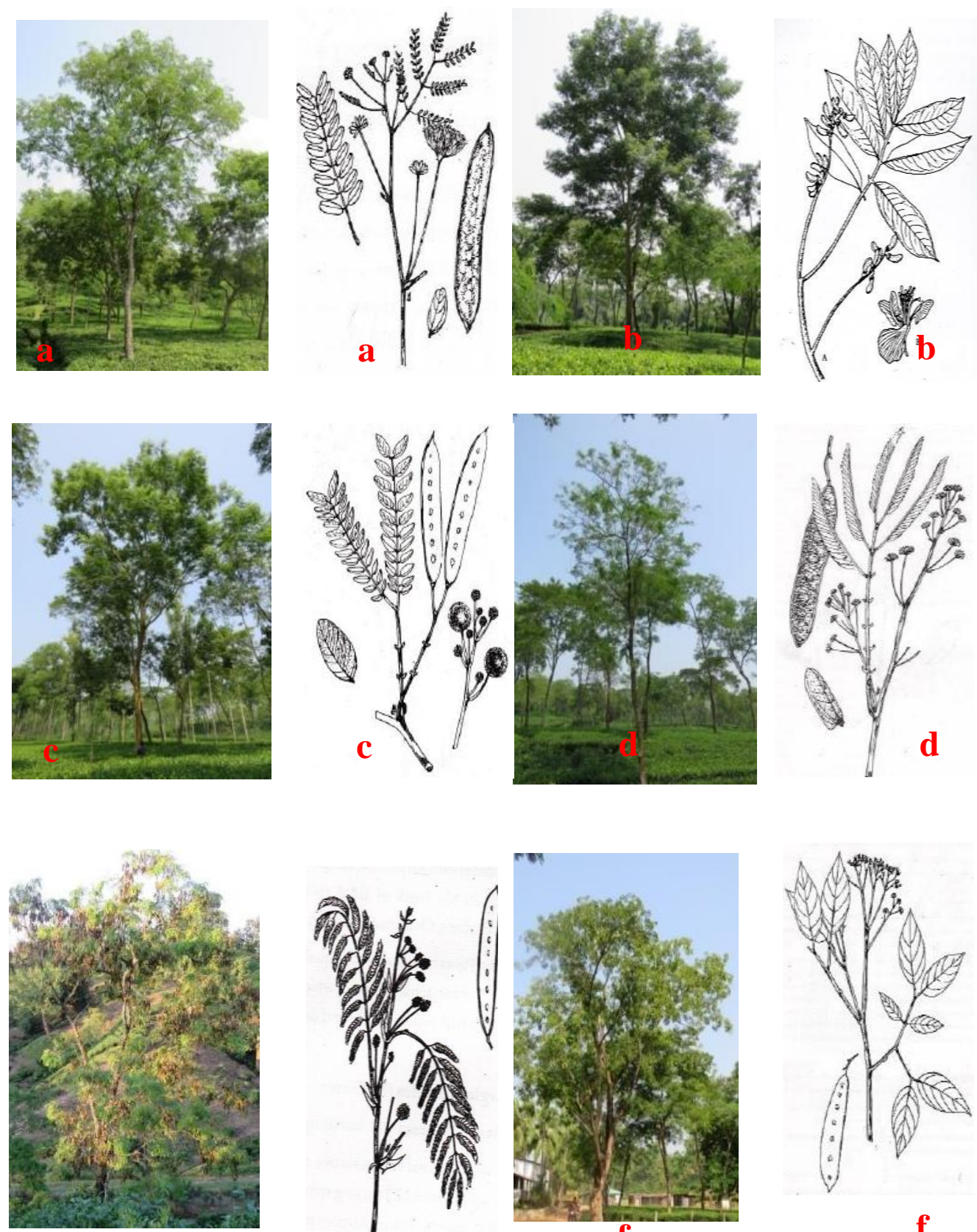

e
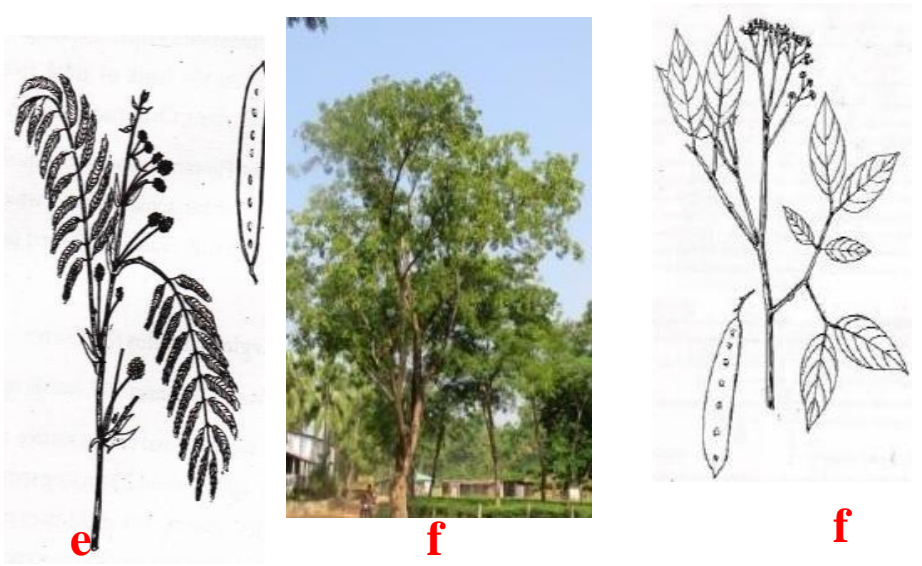

Figure 3. Morphologicaland leaf characteristics of different types of shade trees at investigated tea gardens in Bangladesh. a, b, c, d, e and f indicates Albizia lebbeck, Derris robusta, A. procera, A. odoratissima, A. chinensis and A. lucidor. 

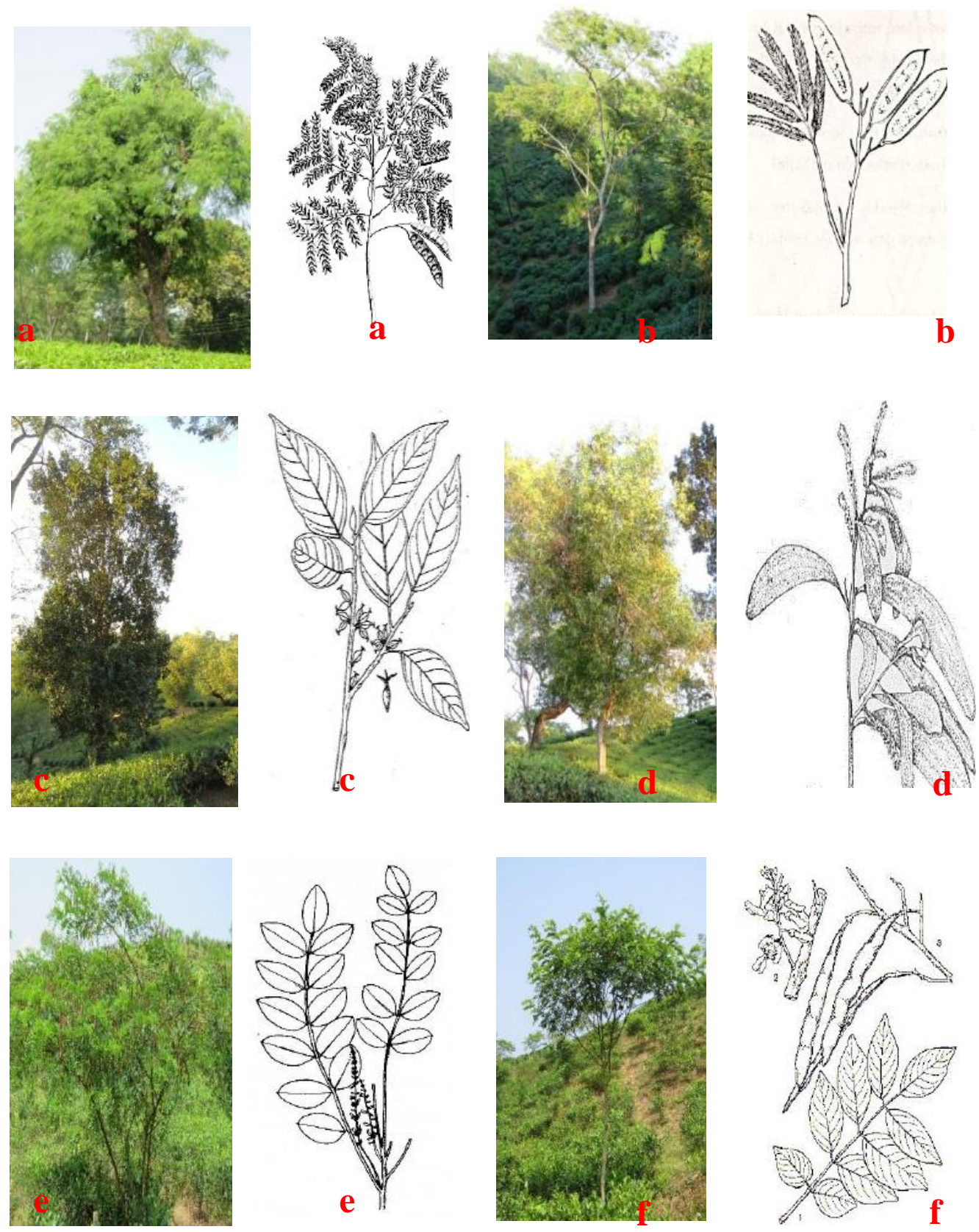

Figure 4. Morphological and leaf characteristics of different types of shade trees at investigated tea gardens in Bangladesh. a, b, c, d, e and f indicates A. moluccana, $A$. richardiana, Artocarpus chaplasha, A. hybrid, Indigofera teysmanniiand Gliricidia sepium. 
Apocynaceae, Moraceae, Protaceae, Lythraceae, Anacardiaceae, and Phyllanthaceae shows $3.22 \%$ generic components of the entire tree flora of the tea plantations areas (Fig.2). The popular permanent shade trees among the tea gardeners are $a$. odoratissima, A. chinensis, A. lebbeck, A. lucidior, A. procera, and D. robusta (Fig. 3 and 4).Indigofera teysmannii is frequently using as a temporary shade species in all investigated tea gardens.Cajanus cajan, Tephrosia candida, Tephrosia candida, Gliricidia sepium, Erythrina lithosperma and Desmodium gyroides species are also used as temporary shade trees in many tea gardens. This finding shows that Fabaceae is the dominant family in all investigated tea garden of Bangladesh. These types of shade trees not only provide shade to tea plants but also helps in replenishing nitrogen loss and controls insect pest due to biopesticide properties of the tree (Pangging and Mandal, 2017; Ahmed et al., 1993). In a similar study, Chowdhury et al. (2015) were enlisted 45 species of angiosperm representing 34 genera of 15 families of shade trees in the tea garden of West Bengal, India. In their assessment, the Leguminosae family showed the highest number of shade trees comprising 13 genera and 22 species and the most dominant shade tree species were Albizia odoratissima, Albizia chinensis, Albizia lebbeck, Albizia procera, Erythrina indica, Dalbergia sissoo,and Melia azedarach.Likewise, Pangging and Mandal (2017) investigated shade tree species and socioeconomic status around the Banderdewa forest range, Arunachal Pradesh, India. The common shade trees found in tea estate around the Banderdewa forest range, Arunachal Pradesh was Melia azedarach L., Albizia procera (Roxb.) Benth. and Albizia lebbeck (L.) Benth. Fabaceae was the most dominant family. Mulugeta (2017) reported that Albizia chinensis, Aleurites fordii and Calophyllum elantus species can be used as shade trees for the successful establishment of a new tea plantation.

\section{CONCLUSION}

A total of 44 species of Angiosperm representing 31 genera of 9 families was enlisted at fifteen tea estates of Bangladesh. Among the estates, Fabaceae family shows the highest number of shade trees comprising 19 genera and 31 species. Further investigations, however, are required to find out the appropriate shade tree species on the growth and yield of tea plants and improvement of quality of made tea.

\section{ACKNOWLEDGMENTS}

The research was supported by the NATP (Phase-II) sub-project of the Bangladesh Agricultural Research Council (BARC), Farmgate, Dhaka, Bangladesh. We are grateful to all estate management persons for their cooperation during the study. Special thanks are due to Forest Botany Division, Bangladesh Forest Research Institute, Chattogram, Bangladesh for their kind assistance in identifying plant species. 


\section{REFERENCES}

Ahmed, Z.U., Begum, Z.N.T., Hassan, M.A., Khondker, M., Kabir, S.M.H., Ahmad, M., Ahmed, A.T.A., Rahman, A.K.A. and Haque, E.U. (2009). Encyclopedia of Flora and Fauna of Bangladesh. Angiosperms. Vols. 5-12. Asiatic Society of Bangladesh.

Ahmed, M., Haq, M., Haq, M.I. and Ali, M.A. (1993). Influence of shade on the incidence of pests in Tea plantation in Bangladesh. Proc. Of the Tea Science \& Human Health. TEATECH 1993, TRI, Kolkata, India. Pp. 326-329.

Barua, D.N. (1979). Effect of long term application of nitrogen fertilizer on yield of tea. Two and $a \mathrm{Bud}, 37: 1-3$.

Brandis, D. (1906). Indian Trees, Bishen Singh Mahendra Pal Singh, Dehradun. Pp. 767.

BTB. (2019). Monthly Bulletin of Statistics on tea. January 2019. Bangladesh Tea Board, Baizid Bostami Road, Nasirabad, Chittagong.

Chowdhury, A., Sarkar, S., Roy, P., Mondal S. and Chowdhury, M. (2015). Inventory of shade trees in Tea gardens of Sub-Himalayans region of West Bengal, India. The International Journal of Science and Technology, 3 (12): 164-168.

FAO. (1987). Nitrogen fixing trees. Regional office for Asia and the pacific. Food and agricultural organization of the United Nations. Bangkok, Pp. 61-70.

Heining, J.D. (1925). List of Plants of Chittagong Collectorate and Hill tracts. Darjeeling, India.

Hooker, J.D. (1872-1897). Flora of British India. Vol. 1-7, Reeve and Co. London.

ITC. (2015). Annual Bulletin of Statistics (September 2015). International Tea Committee. London, U.K. Pp. 158.

Kalita, M.R., Das, K.A. and Nath, J.A. (2014). Comparative study on growth performance of two shade trees in tea agro forestry system. Journal of Environmental Biology, 35: 699702.

Kanjilal, U.N., Das, A., Kanjilal, P.C. and De. R.N. (1939). Flora of Assam, vol.3, A Von Book Co., Delhi, India, Pp.578.

Kanjilal, U.N., Das, A., Kanjilal, P.C. and De. R.N. (1940), Flora of Assam, vol.4, A Von Book Co., Delhi, India, Pp. 377.

Ku, K.M., Choi, J.N., Kim, J., Kim, J.K., Yoo, L.G. and Lee, S.J. (2010). Metabolomics analysis reveals the compositional differences of shade grown tea (Camellia sinensis L.). Journal of Agricultural and Food Chemistry, 58: 418-426

Mulugeta, G. (2017). Effect of different shade tree species on the growth and yield of China hybrid Tea (Camellia sinensis L.) at Palampur Tea Research Station, H.P., India. Journal of Natural Sciences Research, 7 (4): 15-22.

Pangging, G. and Mandal, S. (2017) Assessment of shade trees and socio-economic condition of the Tea workers: a case study of tea estate around Banderdewa forest range. Bulletin of Arunachal Forest Research, 32(1\&2), 62-65.

Pasha, M.K. and Uddin, S.B. (2013). Dictionary of Plant Names of Bangladesh (Vascular Plants). Janokalyan Prokashani. Chittagong, Dhaka, Bangladesh. 
Prain, D. (1903). Bengal Plants. Vol. 1 \& 2. Govt. press Calcutta.

Rahman, M.A. (2016). An enquiry into the living conditions of tea garden workers of Bangladesh: A case study of Khan Tea estate. BRAC Institute of Governance and Development, BRAC University, Dhaka, Bangladesh. M. A Thesis.

Sana, D.L. (1989). Tea Science. $2^{\text {nd }}$ Edition, Ashrafia Boi Ghar, Bangla Bazar, Dhaka, Bangladesh, Pp. 7-81.

Wang, Y.S., Gao, L.P. and Shan, Y. (2012) Influence of shade on flavonoid biosynthesis in Tea (Camellia sinensisL.). Scientia Horticulturae, 141: 7-16. 